\title{
Laparoscopic Splenectomy for Massive Splenomegaly in a Pediatric Patient
}

\author{
Mariana Borges-Dias, MD \\ Pediatric Surgery Department, Faculty of Medicine, Hospital São João, Porto, Portugal. \\ E-mail: mariana.bg.dias@gmail.com
}

Tiago Tuna, MD

Pediatric Surgery Department, Faculty of Medicine, Hospital São João, Porto, Portugal.

Miguel Madureira, MD

Interventional Radiology Department, Faculty of Medicine, Hospital São João, Porto, Portugal.

Carlos Mariz, MD

Pediatric Surgery Department, Faculty of Medicine, Hospital São João, Porto, Portugal.

Tiago Henriques-Coelho, MD, PhD

Pediatric Surgery Department, Faculty of Medicine, Hospital São João, Porto, Portugal.

José Estevão-Costa, MD, PhD

Pediatric Surgery Department, Faculty of Medicine, Hospital São João, Porto, Portugal.

(c) Mary Ann Liebert, Inc. DOI: 10.1089/vor.2017.0443
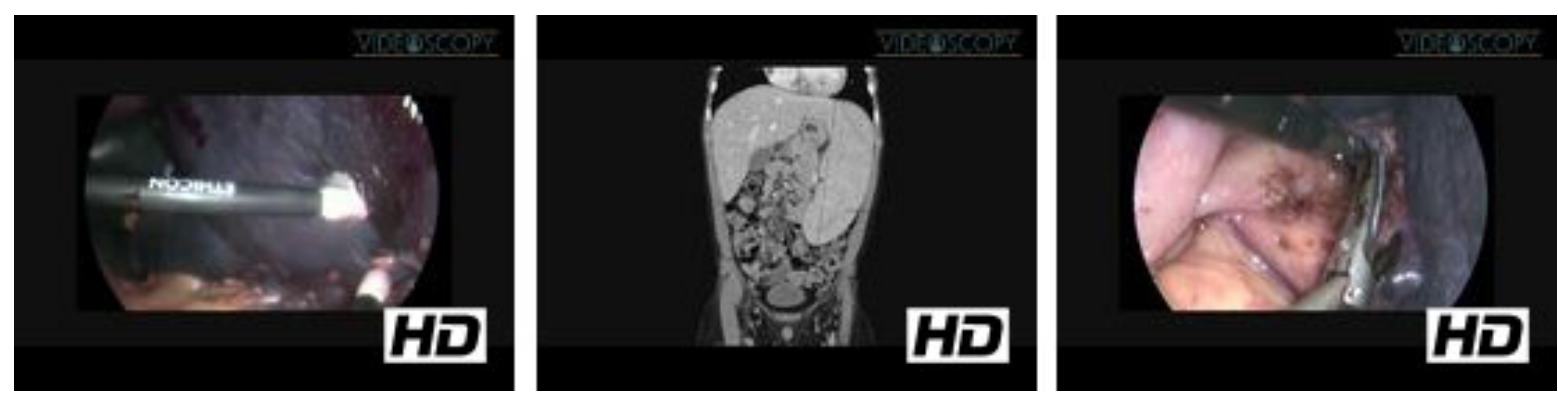

\section{Abstract}

Introduction: Splenectomy is often required in children for various hematologic disorders, although massive splenomegaly is uncommon in pediatric age. Laparoscopic splenectomy for massive splenomegaly is still a controversial and challenging procedure. We present a 5 minutes video of a five-trocar laparoscopic splenectomy of a massive spleen.

Case Report: A 9-year-old male patient with hereditary spherocytosis nonresponsive to medical treatment presented with a massive splenomegaly ( $\sim 18 \mathrm{~cm}$ diameter in tomography). A preoperative splenic artery embolization was performed to allow spleen reduction and to reduce hemorrhagic losses during surgery. A laparoscopic splenectomy using five trocars was performed immediately after the embolization (operative time-195 minutes). An Endo-Cameleon ${ }^{\circledR}$ camera with a view range from $0^{\circ}$ to $120^{\circ}$ was used and revealed to be crucial for important steps during the procedure, namely posterior and superior splenic dissection. Vascular control was obtained using several devices: mechanical stapler, Hem-o-lok ${ }^{\circledR}$, and harmonic scalpel. The massive spleen was mobilized using blunt tip dissectors. The spleen was collected using a $15 \mathrm{~mm}$ endobag $(1500 \mathrm{~mL})$ and removed after extensive manual morcellation. Postoperative time was uneventful, and the patient was discharged on day 2. After 9 months of follow-up he was asymptomatic with favorable cosmetic appearance. 
Conclusions: Several approaches had been developed to deal with massive splenomegaly depending on the background disease. Pitfalls such as small cavity size and significant spleen enlargement were overcome by this perioperative arterial embolization, five-trocar technique, and a multidirection scope.

The corresponding author has no conflicts of interest to disclose of financial or other relationships with companies or organizations that are stakeholders in the topic of the video. There were no sources of funding that could have influenced the outcome of this work. The corresponding author signed this statement on behalf of all coauthors to indicate that the mentioned information is true, correct, and complete.

Runtime of video: 5 mins 7 secs

Keywords: massive splenomegaly, laparoscopic splenectomy, children, hereditary spherocytosis

\section{Cite this video}

Mariana Borges-Dias, Tiago Tuna, Miguel Madureira, Carlos Mariz, Tiago Henriques-Coelho, José Estevão-Costa, Laparoscopic Splenectomy for Massive Splenomegaly in a Pediatric Patient, Videoscopy. 2017, DOI: 10.1089/vor.2017.0443.

Original Publication Date: 2017 